\title{
Research And Application of Online Rate Promotion on General Packet Radio Service in The Online Charging System
}

\author{
Ying Wen ${ }^{1, ~ a ~}$, ChangXiao He $\mathrm{H}^{1, \mathrm{~b}}$, Tao Wang ${ }^{1, \mathrm{c}}$, JiaJia Zhou ${ }^{1, \mathrm{~d}}$, JiaYi Huang ${ }^{1, \mathrm{e}}$, \\ Jiang Yang ${ }^{1, f}$ \\ ${ }^{1}$ China Mobile Group GuangXi Company Limited, Nanning, China \\ wenying@gx.chinamobile.com, bhechangxiao@gx.chinamobile.com, \\ cwangtao@gx.chinamobile.com, ${ }^{d}$ zhoujiajia@gx.chinamobile.com, \\ ehuangjiayi@gx.chinamobile.com, yangjiang@gx.chinamobile.com
}

Keywords: GPRS (General Packet Radio Service), Online Charging System (OCS), Online Rate Abstract. Based on Online Charging System in one communication operator, the paper analyses some reasons for low online rate of General Packet Radio Service (GPRS) in this system. It is out of balance load and has low resource utilization. Some optimization schemes are proposed in this paper. It optimizes the process of the front, dispatch and inverse count module. It adds junction proxy cluster to ensure load balancing. The ratio of receive to response is optimized in the front. The zero fee Charge Detail Records (CDRs) and signaling CDRs in the GPRS are distinguished with special processing. By comparing system performance, the data shows that the optimization module performance is much better than the old one in processing ability and efficiency. It assures load balancing and takes up less host resource and storage space. Online rate is significantly improved and the remind delay is greatly be shortened. It is effective in energy saving and cost reducing on operation and maintenance.

\section{Background}

At present, there are two types in telecommunication, namely online charging and offline charging. Offline charging, namely quasi-realtime charging, is to use firstly and bill later. The billing system doesn't take part in when customers are using telecommunication services. It is charged later according offline CDRs (Charge Detail Records).Therefore it will cause varying degrees arrearages. Online charging can get bill information and go into effect in real time. The feature is that the billing system will take part in the whole process when customers are using telecommunication services. The billing system directly interacts with session control and service control. It is to use and bill at the same time. Therefore it will have much less arrearages and much better charging timeliness.

In 4 Generation (4G, like TDD-LTE) network, customers can get much faster data flow speed and with higher risk of arrearages at the same time. The Online Charging can bill and remind in real time. Based on standard DCC protocol, the OCS in one telecommunication operator communicates with online equipment such as SCP, GGSN and so on. It can count and inversely count according bill information like data flow service. And it can control, bill and remind in real time with telephone traffic control $^{[1-3]}$. After OCS on line, Online rates of many provinces (Online rate equals the ratio of online data flow service quantity to total data flow service quantity) are on the low side. There are varying degrees differences and relate with many modules. A much better suggested scheme isn't provided because of some complicated questions. OCS was been on line in GuangXi province since 2013. The online rate is low. For example, it was about $77 \%$ in 2015 January and February. The remind delay of data flow service and charging delay didn't effectively improved ${ }^{[4]}$. There was a wide gap between several excellent provinces about $90 \%$ to $95 \%$.

\section{The analysis on online charging of mobile data flow service}

Presently the GPRS (or mobile data flow service) of OCS mainly consist of three modules, like front (TS1), dispatch (TB), inverse count(TS2), as shown in Fig.1: 


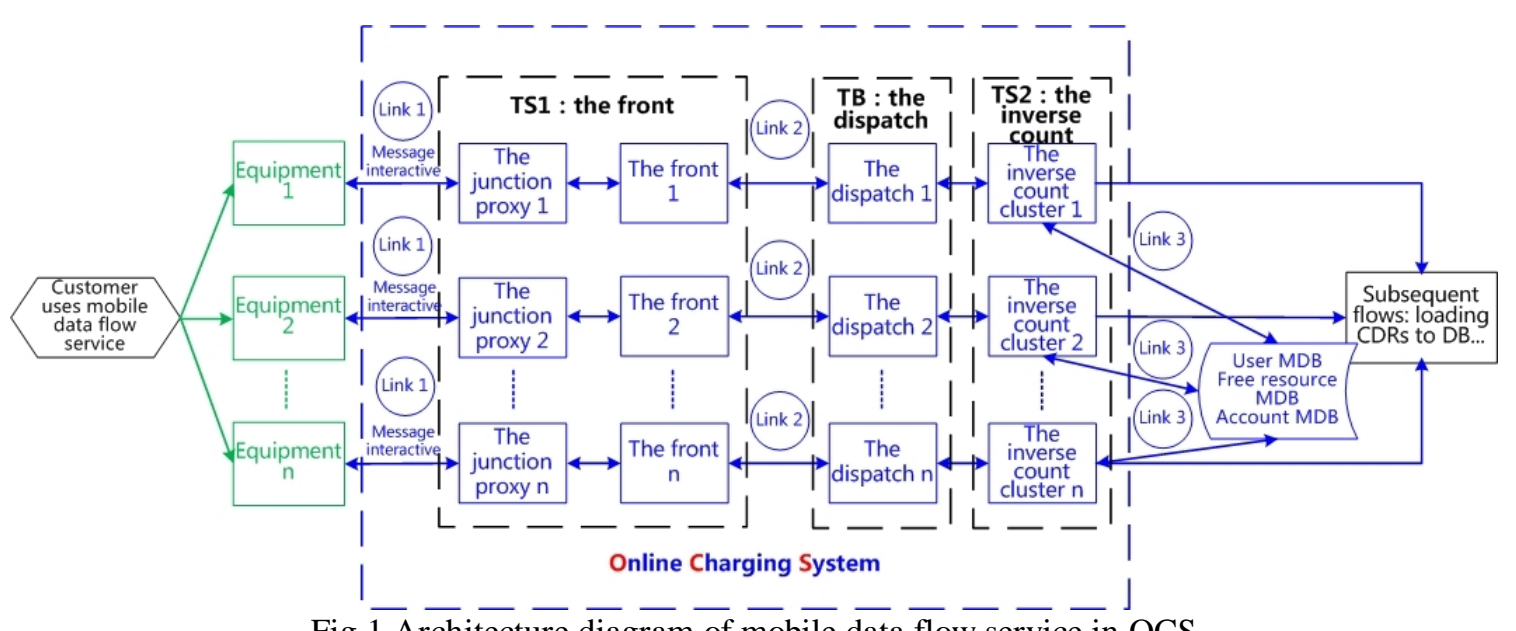

Fig. $\overline{1}$ Architecture diagram of mobile data flow service in OCS

Link 1: The front (TS1) consists of junction proxy and the front. The equipments record data flow and interact with junction proxy through message.

Link 2: The front (TS1) receives message from equipments and response message from the inverse count (TS2). Then it will submit to the dispatch (TB) to schedule and process.

Link 3: The inverse count (TS2) queries some properties of customers, such as online, arrearages, offline. After counting and inversely counting, it reserves customers' application for fee or resource. Then it returns authentication result and customers' application for resource to the junction proxy.

\section{Main problems}

There were about 40 pairs of data about online rate and remind delay from 2015 January to February. Make scatter diagram as shown in Fig.2:

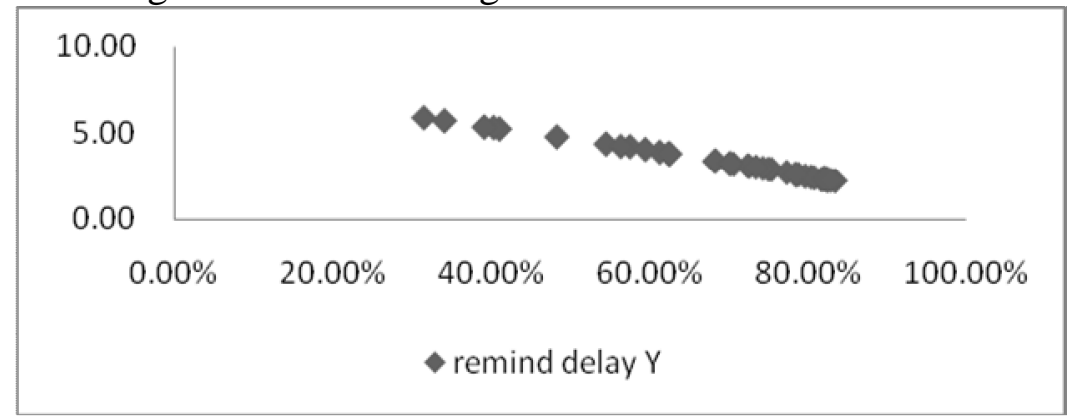

Fig. 2 online rate and remind delay from 2015 Jan. to Feb.

critical correlation coefficient $\gamma_{0.05}=0.312, L_{X X}=\sum X^{2}-\frac{\left(\sum X\right)^{2}}{N}=0.9$,

$L_{Y Y}=\sum Y^{2}-\frac{\left(\sum Y\right)^{2}}{N}=6.7, L_{X Y}=\sum X Y-\frac{\left(\sum X\right)\left(\sum Y\right)}{N}=-6.3$,

correlation coefficient $\gamma=\frac{L_{X Y}}{\sqrt{L_{X X}} \sqrt{L_{Y Y}}}=-0.988,|\gamma|=0.988>0.312$, there was strong negative

correlation between online rate and remind delay. As the online rate increased, the remind delay decreased obviously. If we could promote online rate, we could short bill delay of using the mobile GPRS in province, thus short the remind delay.

Based on the above-mentioned flow, there were four main reasons for low online rate according to statistical analysis as follow:

Business load was unbalanced.

The front (TS1) consists of junction proxy and the front. The junction proxy interacts directly with equipments. Because data flow load were unbalanced on equipments, we couldn't balance load from the service start. The ratio of equipments to front (TS1), dispatch (TB), inverse count (TS2) was 1:1:1:10. Only on equipment load was balanced in the inverse count (TS2). And we couldn't balance 
load in the front (TS1). Therefore, there were large load in some hosts and unbalanced. For example, the business load of many junction proxy hosts and inverse count hosts exceeded the average mean to $1 \pm 5 \%$.

The system load was much high in the front (TS1).

In the front (TS1), the ratio of threads in the receive to the response was 1:4. When the volume of business increased, the system load increased in the front (TS1). It would cause hosts interacted with each other with delay.

The zero fee query occupied much system resource.

Presently CDRs of mobile GPRS were about 1.3 billion. The zero fee CDRs were about up to $85 \%$, namely 1.1 billion. The zero fee CDRs didn't contain fee information. But it would still schedule arrearage account query and charge off module to process zero fee CDRs in the inverse count (TS2). This flow was similar to other non-zero fee CDRs. Each zero fee CDR would query account Memory Data Base (MDB). And it consumed about 100 to $150 \mathrm{~ms}$. Therefore, it would waste much system resource.

The signaling flow CDRs occupied much system resource.

Presently CDRs of mobile GPRS were about 1.3 billion. The signaling flow CDRs were about up to $35 \%$, namely 4.6billion. The signaling flow CDRs didn't contain bill information. But signaling flow CDRs were still passed into the dispatch (TB) and the inverse count (TS2) to process. This flow was similar to other CDRs. Each signaling flow CDR consumed about $500 \mathrm{~ms}$. Therefore, it would waste much system resource.

\section{The Optimized Measures}

Based on the above-mentioned problems, there were four main optimized measures.

To balance business load, the process of CDRs was optimized and junction proxy cluster was used.

A CDRs process method with balancing efficiency and balance was applied. All equipment accessed to junction proxy cluster to dispatch evenly to the front (TS1). Therefore, all business load from equipment could be balanced in the inverse count (TS2). And it ensured to balance business load in the system.

To reduce waste, the thread of receive to response ratio in the front (TS1) and the dispatch (TB) were optimized.

Some relative logs of receive message and response message in the front (TS1) were analyzed. It showed response in the front (TS1) was much idler. We got a best configuration scheme by using orthogonal experimental. The thread of receive to response ratio in the front (TS1) was changed from 1:4 to $2: 3$. This scheme promoted interaction efficiency and online rate.

The main steps of orthogonal experimental were as follows:

(1) The purpose of experiment: based on technical principle, online rate were affected by 3 factors in the OCS: receive in the front (TS1), response in the front (TS1) and dispatch in the inverse count (TS2). We used orthogonal experimental to choose the best configuration and promoted system efficiency.

(2) The index of observation: online rate (\%)

(3) Choose factor and level, make factor and level table

Based on suggested factor configuration and system operation experience, we make factor and level table as shown in Table 1.

Table 1 The factor and level table

\begin{tabular}{rccc}
\hline Factor & $\begin{array}{c}\text { Thread of receive } \\
\text { in the front (TS1) }\end{array}$ & $\begin{array}{c}\text { Thread of response } \\
\text { in the front (TS1) }\end{array}$ & $\begin{array}{c}\text { Thread in the } \\
\text { dispatch (TB) }\end{array}$ \\
\hline Level 1 & 1 & 4 & 1 \\
\hline Level 2 & 2 & 3 & 2 \\
\hline
\end{tabular}


(4) Implement experimental scheme

We selected L4 (23) orthogonal experimental and work out experimental scheme. According to defined terms, we did experiment and checked result as shown in Table 2:

Table 2 The $\mathrm{L}_{4}\left(2^{3}\right)$ table

\begin{tabular}{|c|c|c|c|c|}
\hline \multirow[t]{2}{*}{$\begin{array}{r}\text { Factor } \\
\text { Column No. }\end{array}$} & $\begin{array}{c}\text { The receive in the } \\
\text { front (TS1) A }\end{array}$ & $\begin{array}{c}\text { The Response in the } \\
\text { front (TS1) B }\end{array}$ & $\begin{array}{c}\text { The dispatch (TB) } \\
\text { C }\end{array}$ & \multirow[t]{2}{*}{ Online rate $(\%)$} \\
\hline & 1 & 2 & 3 & \\
\hline 1 & $1(1)$ & $1(4)$ & $1(1)$ & 84.33 \\
\hline 2 & 2(2) & $1(4)$ & 2(2) & 87.83 \\
\hline 3 & $1(1)$ & $2(3)$ & $2(2)$ & 83.37 \\
\hline 4 & $2(2)$ & $2(3)$ & 1（1） & 90.25 \\
\hline Sum of Level I & 167.7 & 172.16 & 174.58 & \multirow{3}{*}{ Grand total $=1037.34$} \\
\hline Sum of Level II & 178.08 & 173.62 & 171.2 & \\
\hline Range $\mathbf{R}$ & 10.38 & 1.46 & 3.38 & \\
\hline
\end{tabular}

(5) Analyzed experiment result

1) Took a look: Table 2 showed the result of No. 4 experiment was best. Therefore, A2B2C1 was a better scheme.

2) Took a count: Different experiments had varying online rate. By counting level, range and mean value, we figured out a better scheme, namely A2B2C1.

3) We obtained primary and secondary relationship according to range $\mathrm{R}$ : $\mathrm{A}-\mathrm{C}-\mathrm{B}$.

4) We did tracing test to verify the validity of the scheme. We applied the result of orthogonal experimental to the whole province in May $25^{\text {th }}$ to $28^{\text {th }}$. The online rate was obviously promoted and up to $92.6 \%$.

Conclusions as a result, A2B2C1 is a better scheme as shown in Table 3.

Table 3 A better scheme

\begin{tabular}{cccc}
\hline Factor & $\begin{array}{l}\text { Thread of receive } \\
\text { in the front (TS1) }\end{array}$ & $\begin{array}{c}\text { Thread of response } \\
\text { in the front (TS1) }\end{array}$ & $\begin{array}{c}\text { Thread in the } \\
\text { dispatch (TB) }\end{array}$ \\
\hline Value & 2 & 3 & 1 \\
\hline
\end{tabular}

To reduce waste, the system didn't dispatch arrearage account query and charge off module when processing the zero fee CDRs.

The zero fee CDRs were about up to $85 \%$, namely 1.1 billion. And they didn't contain fee information. The system added a judge and didn't dispatch arrearage account query and charge off module when processing theses CDRs.

To reduce waste, the system didn't charge signaling flow CDRs.

The signaling flow CDRs were about up to 35\%, namely 4.6billion. And they didn't contain bill information. The system added a judge according to service code in the inverse count (TS2). If it was a signaling flow CDR with signaling service code, it would be process as error CDR and wouldn't be sent to subsequent link to reduce system resource waste.

\section{The optimization effect}

Before optimization, the mean CPU utilization ratio and memory utilization ratio of the reverse hosts were up to $83.2 \%$ and $83.1 \%$ in Mar. 2015. After optimization, all equipment accessed to junction proxy cluster to dispatch evenly to the front (TS1). Therefore, it ensured to balance business load in the system. The above utilization ratios were reduced to 35.8\% and 51.2\% in Sep. 2015.

Before optimization, the mean response delay was 500ms in Mar. 2015. After optimization, the process logic was adjusted to promote response efficiency in the front (TS1) and in the dispatch (TB). The mean response delay was reduced to $150 \mathrm{~ms}$ in Sep. 2015.And the response speed was promoted about $30 \%$. 
After optimization, the system didn't dispatch arrearage account query and charge off module when processing zero fee CDRs. The mean response delay was reduced from 150ms to 90ms in Sep. 2015. And the response speed was promoted about $20 \%$.

After optimization, the system processed signaling flow CDRs as error CDRs. The business load was reduced about $35 \%$. The mean response delay was reduced from $90 \mathrm{~ms}$ to $80 \mathrm{~ms}$ in Sep. 2015. And the response speed was promoted about $11 \%$. Meanwhile, it reduced about 4.6billion CDRs and saved about 180TB storage space.

Before optimization, online rate of mobile data flow service was about 77.4\% in Mar. 2015.After optimization, it was obviously promoted to $97.6 \%$ in Sep. 2015 . The online rate came up to advanced province standards and ranked top nationally. The online rate was as shown in Table 4.

Table 4 The online rate of mobile data flow service

\begin{tabular}{cccccccc}
\hline Date & $\mathbf{2 0 1 5 0 3}$ & $\mathbf{2 0 1 5 0 4}$ & $\mathbf{2 0 1 5 0 5}$ & $\mathbf{2 0 1 5 0 6}$ & $\mathbf{2 0 1 5 0 7}$ & $\mathbf{2 0 1 5 0 8}$ & $\mathbf{2 0 1 5 0 9}$ \\
\hline Oneline rate & $77.4 \%$ & $80.4 \%$ & $83.2 \%$ & $90.7 \%$ & $93.1 \%$ & $97.5 \%$ & $97.6 \%$ \\
\hline
\end{tabular}

Before optimization, the ratio of complaints about remind delay was about 9\% in Mar. 2015. After optimization, it was reduced obviously to $2.1 \%$ in Sep. 2015 as shown in Table 5.

Table 5 The ratio of complaints about remind delay of mobile data flow service

\begin{tabular}{cccccccc}
\hline Date & $\mathbf{2 0 1 5 0 3}$ & $\mathbf{2 0 1 5 0 4}$ & $\mathbf{2 0 1 5 0 5}$ & $\mathbf{2 0 1 5 0 6}$ & $\mathbf{2 0 1 5 0 7}$ & $\mathbf{2 0 1 5 0 8}$ & $\mathbf{2 0 1 5 0 9}$ \\
\hline $\begin{array}{l}\text { the ratio of complaints } \\
\text { about remind delay }\end{array}$ & $9.0 \%$ & $7.6 \%$ & $6.9 \%$ & $5.3 \%$ & $4.1 \%$ & $2.3 \%$ & $2.1 \%$ \\
\hline
\end{tabular}

After applying above optimization, it saved host resource and reduced expansion about 6 X86 hosts. It was about 0.26 million per X86 host. According to this, it saved host cost about 156 million. It saved about $180 \mathrm{~TB}$ storage space. According to 0.15 million per 40TB, it saved storage cost about 68 million. At the same time, it charged and control in real time. It could promote online rate obviously and short remind delay. It reduced the risk of arrearage and revenue loss. And it was helpful to promote the degree of customers' satisfaction. The data showed that the arrearage of mobile data flow was reduced about $10 \%$ and the arrearage was reduced about 750million from Mar. to Sep. in 2015.

\section{Conclusions and prospect}

This paper adjusts and adapts the front (TS1), the dispatch (TB) and the inverse count (TS2). It balances the whole business load and promotes obviously. Therefore, it reduces system overhead, saves host resource and storage space resource. It is effective in energy saving and cost reducing on operation and maintenance. And it gives strong support to vigorously develop data flow in $4 \mathrm{G}$ network. Then we can consider how to realize charging in time in national roaming mobile data flow service and other value added service, such as monternet sms (like weather forecast), monternet mms (like mobile phone newspaper), WAP (like AND reading), KJAVA (like AND game) and so on. We can charge these service in time and reduce remind delay, thus reducing the risk of arrearage and revenue loss.

\section{Reference}

[1] NGBOSS2-BOSS (V4.5) Online Charging System interface specification v1.1.0, 2013

[2] NGBOSS2-BOSS (V4.5) service specification, 2013

[3] Haiqiang Zhang. The Design and Realization of Online Charging System in Unicom Company, Xiamen University, 2014

[4] Ying Wen, Changxiao He, Tianchun Ju. The Optimization And Application of Threshold Remind Short Message Delay on Mobile Data Flow Service. Telecom Engineering Techniques and

Standardization, 2015(5), p. 18-22 